\title{
Contactless Wide-Band Nearfield Microwave Sensing Techniques in Microfluidic Applications
}

\author{
$\underline{\text { T. Nacke }}^{1}$; A. Barthel'; B.P. Cahill'; R. Klukas ${ }^{2}$; M. Meister ${ }^{1}$; C. Pflieger ${ }^{1}$; D.Beckmann ${ }^{1}$ \\ ${ }^{1}$ Institut für Bioprozess- und Analysenmesstechnik e.V., Rosenhof, \\ 37308 Heilbad Heiligenstadt, Germany \\ thomas.nacke@iba-heiligenstadt.de \\ ${ }^{2}$ IRK Dresden GmbH, Nossener Str. 30, 01723 Mohorn, Germany
}

\begin{abstract}
:
There is a widespread need for highly-sensitive robust sensors for process measurements in microfluidics that have no direct contact to the fluid. This contribution describes how the measurement of the variation of the dielectric properties (conductivity and permittivity) offers an innovative approach to investigating biological and chemical matter and processes in microsystems, for example, cell growth, cell metabolite changes, and the concentration of large aqueous-based molecules. This presentation describes an innovative high frequency sensor that integrates RF/microwave detection in a microfluidic network for quick and precise biological and chemical analysis. It consists of a microstripline waveguide with resonant properties placed adjacent to a microfluidic channel. Contactless conductivity detection is of high interest for many applications in microfluidics and it is particularly attractive for measuring the concentration of inorganic and organic ions and matter that are not directly accessible by optical means.
\end{abstract}

Key words: Microwave spectroscopy, sensor, microfluidic, resonator, stripline

\section{Introduction}

In the last two decades, the advance of microfluidic systems has driven demand for biological and chemical sensor applications. Numerous applications of microfluidics have been developed in chemical, biological and other technical fields [2, 3]. A key requirement for micro-reaction systems is the measurement of process parameters such as conductivity $(\sigma)$, permittivity $(\varepsilon)$, fluorescence, optical density, flow rate data, $\mathrm{pH}$, and dissolved oxygen and in real time. In particular for biosystems, the monitoring of cell density, cell growth and cell viability are very important parameters. The basic metabolic processes occurring within cells determined by biophysical and biochemical variables which are accordance direct by cell growth or by metabolic changes. Microwave measurement technology has opened up a broad range of applications for measuring dielectric materials properties which are suitable for application as contactless nondestructive sensors in microsystems [4]. Previously, we have described the non-contact measurement at RF frequencies of the conductivity of aqueous droplets in segmented flow [5].

\section{Methodology}

Dielectric spectroscopy is a technique based on the dominant role that electrical charges play in molecular interaction with matter. In recent years the rapid development of communications technology has opened up the opportunity to extend dielectric spectroscopy to microwave frequencies, allowing the measurement of the dielectric properties of solid matter and liquids over the frequency range from $100 \mathrm{MHz}$ to $100 \mathrm{GHz}$. Relaxation phenomena with characteristic time constants between fs and some $\mu s$ are accessible to practical applications. The basic set-up of a microwave reflection sensor is presented in Fig. 1.

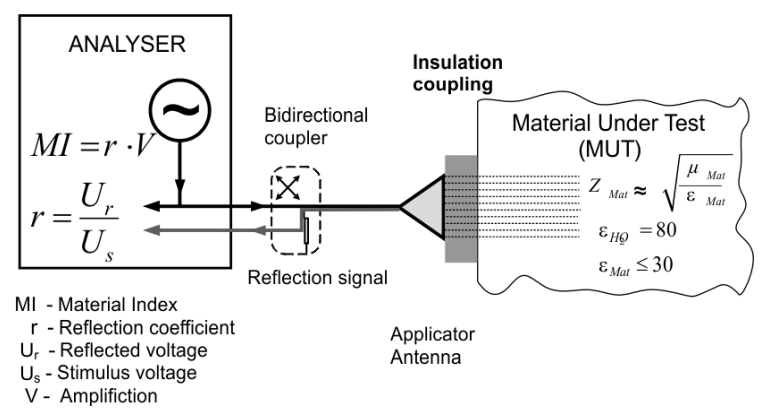

Fig. 1. Basics of microwave reflection measurements 
Although microwave sensors are state of the art, the application and combination of liquids and electromagnetic waves, especially at the millimeter and micrometer scale, is not common. One additional advantage of measurement at microwave frequencies, in comparison with that at lower frequencies $(f)$, is that the DC conductivity $(\sigma)$ effects on material properties (permittivity $\varepsilon$ ) can be neglected. Fig. 2 shows the real part $\varepsilon^{\prime}$ and negative imaginary part $\varepsilon "$ of the extended Debye relaxation function (1) of an aqueous liquid. The dielectric spectrum can be well described by a Debye-type relaxation function with an additional term for the saline dependence (simplified) [6]:

$$
\varepsilon=\varepsilon_{(\infty)}+\frac{\varepsilon_{(0)}-\varepsilon_{(\infty)}}{1+j \omega \tau}-\frac{j \sigma}{\varepsilon_{0} \omega}
$$

where $\tau$ is the relaxation time of the water dipole, $\omega=2 \pi f, \varepsilon_{(\infty)}$ the permittivity at high frequency, $\varepsilon_{(0)}$ the permittivity at low frequency and $\varepsilon_{0}$ the permittivity of free space.

Tab. 1: Relaxation parameters of aqueous solutions with different ionic strengths.

\begin{tabular}{|c|c|c|}
\hline & Solution 1 & Solution 2 \\
\hline$\varepsilon_{(0)}$ & 78.36 & 71.00 \\
\hline$\varepsilon_{(\infty)}$ & 5.40 & 5.40 \\
\hline$\tau_{(\omega)} / p s$ & 8.28 & 8.28 \\
\hline$\sigma / \mathrm{Sm}^{-1}$ & 0.001 & 1.00 \\
\hline$\varepsilon_{0} / \mathrm{VsA}^{-1} \mathrm{~m}^{-1}$ & \multicolumn{2}{|c|}{$8.85419 \mathrm{E}^{-12}$} \\
\hline
\end{tabular}

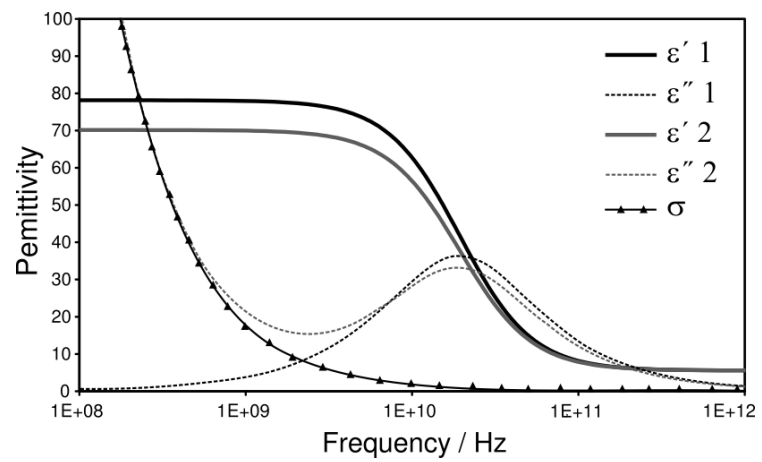

Fig. 2. Complex permittivity of two examples with different conductivity as a function of the frequency. The plotted function $\sigma$ shows the frequency response of the last part of equation $1, \sigma /\left(\varepsilon_{0} \omega\right)$, for solution 2 .

The method is based on the fact that the electrophysical parameters of polar liquids (water and water mixtures) depend on frequency in the microwave range. Changes of water content in a mixed solution produce changes in the resonant frequency. $W$ the width at half maximum and A-area are typically used as measures of the frequency response of a resonator and are shown schematically in Fig. 3 , where the reflection coefficient $\left(S_{11}\right.$ expressed in $\mathrm{dB}$ ) is presented as a function of frequency for liquids varying water content. These electrical properties are the basis of numerous calculations for the determination of the complex permittivity of mixtures or as a calibration function for determining the composition of material compounds.

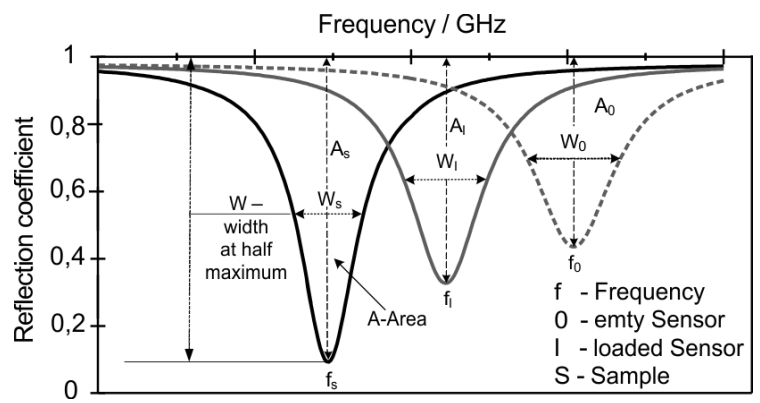

Fig. 3. Frequency response of a resonator, with measured $S_{11}$ of a loaded and unloaded sensor and basics of parameter estimation

It is suggested that readers refer to these resources for a complete description of the relevant theory regarding the interaction of microwaves with dielectric materials $[7,8,9]$. This paper makes use of the following frequency dependent parameters: reflection coefficient $\left(S_{11}\right)$, magnitude, partition of magnitude and phase of the signal. Other parameters that can be used to characterize the system are the change in resonant frequency, the quality factor (Q-factor) and the attenuation, which depends on the load of the resonator sample volume with different matter.

Equations 2 and 3 introduces the factors $k_{l}$ and $k_{2}$ that take into account the geometry of the measurement cell and sensor $[7,8,9]$ :

$$
\begin{aligned}
& \varepsilon_{r}^{\prime}=1+\frac{f_{(0)}-f_{(l)}}{f_{(0)}} \cdot k_{1} \\
& \varepsilon^{\prime \prime}=\left(\frac{1}{\mathrm{Q}_{(l)}}-\frac{1}{\mathrm{Q}_{(0)}}\right) \cdot k_{2}
\end{aligned}
$$

The factors $k_{1}$ and $k_{2}$ can be experimentally determined through calibration with solutions with known values for $\varepsilon^{\prime}$ und $\varepsilon^{\prime \prime}$. In this way, we can account for artifacts that cannot be accounted for by simulation. 


\section{Experimental Procedure}

The experiments were performed with a specially designed and constructed sensor consisting of a stripline waveguide that was integrated in a resonator (stripline in a cavity) in reflection measurement mode (see Fig. 4.). The stripline waveguide is made from two copper cantilevers of $36 \mathrm{~mm}$ length and $3 \mathrm{~mm}$ width. The cantilevers were separated from each other by $3 \mathrm{~mm}$. The stripline waveguide was placed inside an aluminium housing (51 $\mathrm{x}$ $26 \mathrm{~mm}$ ) and connected directly to the soldering terminals of an SMA connector. This geometric configuration resulted in four single resonant peaks in the frequency range up to $10 \mathrm{GHz}$. These single resonant peaks facilitate the wideband determination of the material properties of the fluids under test. A tubing was placed between the stripline, through which the sample fluids could be pumped. Silicon tubing of $3 \mathrm{~mm}$ outside diameter and $2.5 \mathrm{~mm}$ inner diameter was used for the practical measurements.

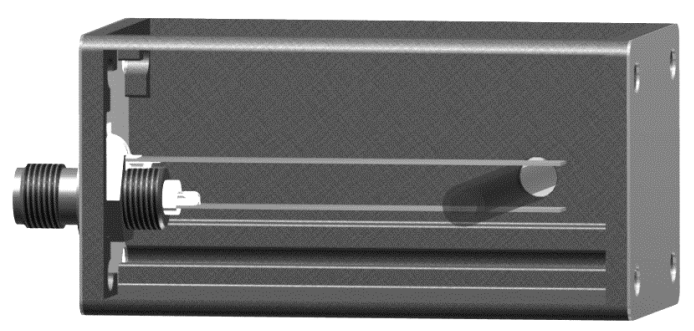

Fig. 4. Schematic view of the microstripline resonator

In order to measure and record the results, the sensor was connected by coaxial cable to a vector analyzer (Anritsu MS MS4644A). All measurements were carried out at constant room temperature.

\section{Experimental Results}

Investigation of the frequency response of the sensor were carried out using air, water and ethanol (see Fig. 5.).

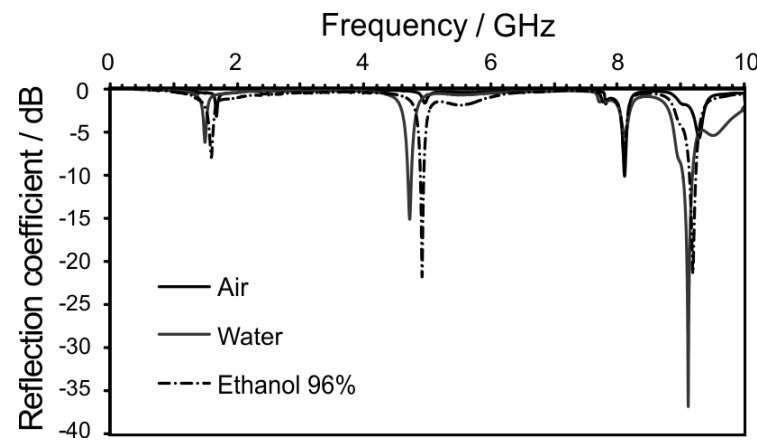

Fig. 5. Frequency response of the microstrip resonator sensor (Fig 4)
In the first experiments the resonant peaks were disturbed by a high oscillation. The exact estimation of the resonant frequency and the width at half maximum was only possible at reduced accuracy and therefore the accuracy of equations 2 and 3 was also adversely affected. For this reason, equation 4 was developed, to improve the calculation of the area underneath a resonant curve (in this equation $\mathrm{Si}_{(\mathrm{S} 11)}$ is a reflection index).

$S I_{\left(S_{11}\right)}=\frac{A_{x}}{f_{0}-f_{x}}$

Figure 6 shows the application of this method of calculation to the measurement of the conductivity of aqueous solutions of various conductivities.

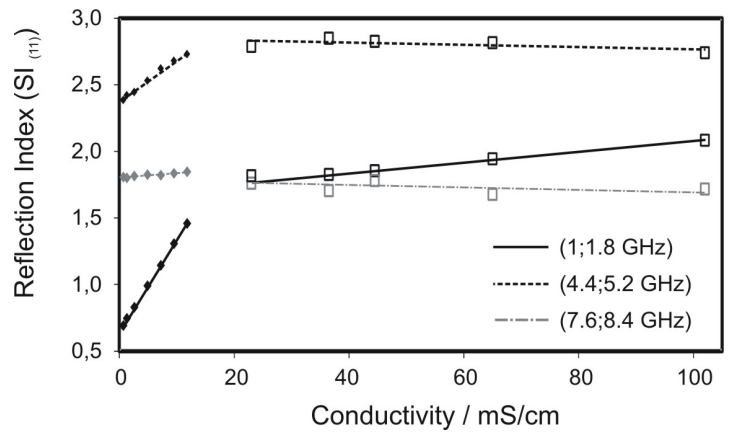

Fig. 6. Measurement of $\mathrm{KCl}$ concentration at three single resonance peaks (see Fig. 5) in the frequency range from 1 to $9 \mathrm{GHz}$

Three separate ranges can be observed: at low conductivities there is the highest sensitivity for conductivity, at high conductivities the salt reaches saturation levels and between these ranges the geometry of the sensor was not optimal for measurement. Currently practical and theoretical work is being carried out to optimise the geometry of the sensor.

The example in Fig. 7 shows the measurement of the alcohol concentration of an aqueous solution with the sensor.

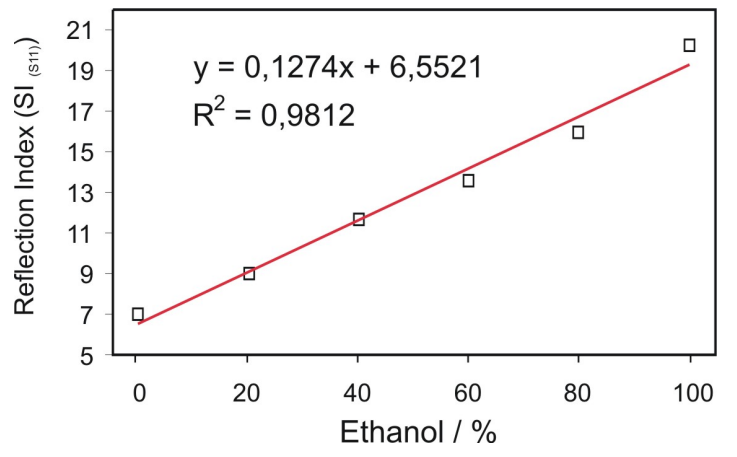

Fig. 7. Reflection index $S I_{(S 11)}$ as a function of the Ethanol-Water dilution. 
The sensing of air bubbles or of phase boundaries is of importance for many applications in microfluidics. For example, in applications of segmented flow, aqueous solutions are embedded in an immiscible carrier liquid [10]. For the analysis of droplet position, speed and volume, the sensing of the presence or absence of the droplet is of great importance. Figure 8 shows an example of the sensing of air bubble detection in an aqueous carrier liquid.

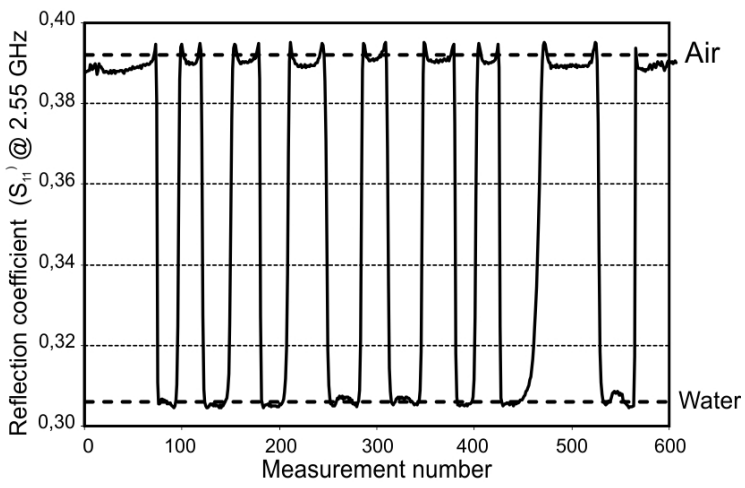

Fig. 8. Injected air bubbles of various volumes in a small water filled tube

\section{Conclusion and future Work}

The next step will be the online determination of the variation of the conductivity and permittivity of droplet based bioreactors in a segmented flow system so that cell growth in the droplets can be measured. The number of cells, the consumption of nutrients by the cells and the direct influence of metabolic processes all affect the passive dielectric properties.

For a suitable sensor design it is possible to provide a plurality or stripline and resonator configurations which can be designed and connected in accordance with the geometry of the object (fluid channels or small reactors) to be monitored. Measurements were performed on different capillary tubes with inner diameters between $500 \mu \mathrm{m}$ and $5 \mathrm{~mm}$.

This technique in combination with reasonablypriced high-frequency electronic circuits opens up the possibility of simplifying automation and miniaturizing routine application for a wide variety of microfluidic applications.

\section{Acknowledgements}

The authors would like to thank the Thuringian Ministry of Education, Science and Culture for financial support.
B. Cahill would like to thank the European Community for financially supporting the Marie Curie ERG project EWETDYNAM under reference number PERG05-GA-2009-247784.

\section{References}

[1] J. Melin, S. R. Quake, Microfluidic large-scale integration: the evolution of design rules for biological automation, Annu. Rev. Biophys. Biomol. Struct. 36: 213-231 (2007); doi: 10.1146/annurev.biophys.36.040306.132646

[2] G.M. Whitesides, The origins and the future of microfluidics. Nature, 442:368-73 (2006); doi: 10.1038/nature05058

[3] G. Pasirayi, V. Auger, et al., Microfluidic bioreactors for cell culturing: A review, Micro and Nanosystems, 3: 137-160 (2011); doi: $10.2174 / 1876402911103020137$

[4] C.R. Song, P.S. Wang, A radio frequency device for measurement of minute dielectric property changes in microfluidic channels, Applied Physics Letters, 94 (2) (2009); doi: 10.1063/1.3072806

[5] B.P. Cahill, R. Land, T. Nacke, M. Min, D. Beckmann, Contactless sensing of the conductivity of aqueous droplets in segmented flow, Sensors \& Actuators: B. Chemical, 159(1) 286-293 (2011); doi: 10.1016/j.snb.2011.07.006

[6] A. Stogryn, G. Desargant, The dielectric properties of brine in sea ice at microwave frequencies, IEEE Transactions on Antennas and Propagation, 33(5) 523-5 $32 \quad$ (1985); doi:10.1109/TAP.1985.1143610

[7] M. Venkatesh, G. Raghavan, An overview of dielectric properties measuring techniques, Canadian Biosystems Engineering 47(7): 15-30. (2005)

[8] K. K. Joshi, I. Allika, et al., Nondestructive Microstrip Resonator Technique for the measurement of moisture / permittivity in crude oil, Proceedings of the XXVIIIth URSI General Assembly in New Delhi (October 2005) 324-330

[9] R. Knöchel, R. Jahns, et al., A Resonator-based Moisture Meter for High Moisture Levels, Proceedings of the First Europeen Conference on Moisture Merasurement, Weimar, Germany, Oct. 5-7: 53-62. (2010)

[10] K. Martin, T. Henkel, V. Baier, A. Grodrian, T. Schön, M. Roth, J.M. Köhler, J. Metze, Generation of larger numbers of separated microbial populations by cultivation in segmented-flow microdevices, Lab on a Chip, 3, 202-207 (2003); doi: 10.1039/B301258C 